\title{
Oh Behave! \\ Agents-Based Behavioral Representations in Problem Solving Environments
}

\author{
M. North ${ }^{1}$, C. Macal, and P. Campbell \\ ${ }^{1}$ Argonne National Laboratory, 9700 S. Cass Avenue, Argonne, IL 60439 \\ \{north, macal, campbell\} @anl.gov
}

\begin{abstract}
The development of deregulated electricity systems around the world has produced the need for simulation systems that are capable of addressing the complexities that arise in the new markets. Agent-based models allow the use of complex adaptive systems approaches that are capable of producing tools or problem solving environments that can address the behavior of each of the participants within the electricity market. The agents in the tools are allowed to establish their own objectives and apply their own decision rules. They can be developed to learn from their previous experiences and change their behavior when future opportunities arise. In this paper, we will argue that the same type of agent-based technology that is used to produce "realistic" agent behavior in agent-based simulation tools at Argonne National Laboratory can also be used to embed these tools in problem solving environments.
\end{abstract}

\section{Introduction}

The development of deregulated electricity systems around the world has produced the need for simulation systems that are capable of addressing the complexities that arise in the new markets. As these electric utility systems continue to evolve from regulated, vertically integrated monopoly structures to open markets that promote competition among suppliers and provide consumers with a choice of services, the unbundling of the generation, transmission, and distribution functions that is part of this evolution creates opportunities for many new players, or agents, to enter the market. It even creates new types of industries, including power brokers, marketers, and load aggregators or consolidators. As a result, fully functioning markets are distinguished by the presence of a large number of companies and players that are in direct competition. Economic theory holds that this will lead to increased economic efficiency expressed in higher quality services and products at lower retail prices. Each market participant has its own unique business strategy, risk preference, and decision model. Decentralized decision-making is one of the key features of the new deregulated markets.

Agent-based models (ABMs) allow the use of complex adaptive systems approaches that are capable of producing tools or problem solving environments (PSE) that can address the behavior of each of the participants within the electricity market. The agents in the tools are allowed to establish their own objectives and apply their own decision rules. They can be developed to learn from their previous experiences and change their behavior when future opportunities arise. 
A PSE is a computer system that provides all the computational facilities needed to solve a target class of problems. These features include advanced solution methods, automatic and semiautomatic selection of solution methods, and ways to easily incorporate novel solution methods. Moreover, PSEs use the language of the target class of problems, so users can run them without specialized knowledge of the underlying computer hardware or software. By exploiting modern technologies such as interactive color graphics, powerful processors, and networks of specialized services, PSEs can track extended problem solving tasks and allow users to review them easily. Overall, they create a framework that is all things to all people: they solve simple or complex problems, support rapid prototyping or detailed analysis, and can be used in introductory education or at the frontiers of science [1].

An agent is a software representation of a decision-making unit. Agents are selfdirected software objects with specific traits and typically exhibit bounded rationality, meaning that they make decisions using limited internal decision rules that depend only on imperfect local information. Emergent behavior is a key feature of ABMs. Emergent behavior occurs when the behavior of a system is more complicated than the simple sum of the behaviors of its components [2].

In this paper, we will argue that the same type of agent-based technology that is used to produce "realistic" agent behavior in the Electricity Market Complex Adaptive Systems model (EMCAS), and other agent-based simulation tools at Argonne National Laboratory (ANL), can also be used to embed these tools in a PSE-type environment - i.e. one in which all the intricacies of the underlying computer hardware and software are hidden from the user, who is then free to focus on modeling meaningful solutions.

\section{EMCAS}

EMCAS is an electricity market model related to several earlier models $[3,4]$. EMCAS includes a large number of different agents to model the full range of time scales - from hours to decades - that are needed to understand the domain [5]. The focus of agent rules in EMCAS varies to match the time continuum, as shown in Fig. 1. Over longer time scales, human economic decisions dominate. Over shorter time scales, physical laws dominate. Many EMCAS agents are relatively complex, or "thick," compared to typical agents. EMCAS agents are highly specialized to perform diverse tasks, ranging from acting as generation companies to modeling transmission lines, as shown in Fig. 2. To support specialization, EMCAS agents include large numbers of highly specific rules. EMCAS agent strategies are highly programmable. Users can easily define new strategies to be used for EMCAS agents and then examine the marketplace consequences of these strategies. EMCAS and its component agents are currently being subjected to rigorous quantitative validation and calibration. 
Decision Level 1 Real Time Dispatch

Decision Level 2

Day Ahead Planning

Decision Level 3

Week Ahead Planning

Decision Level 4

Month Ahead Planning

Decision Level 5

Year Ahead Planning

Decision Level 6

Medium to Long-Term

Planning (2-10 years)

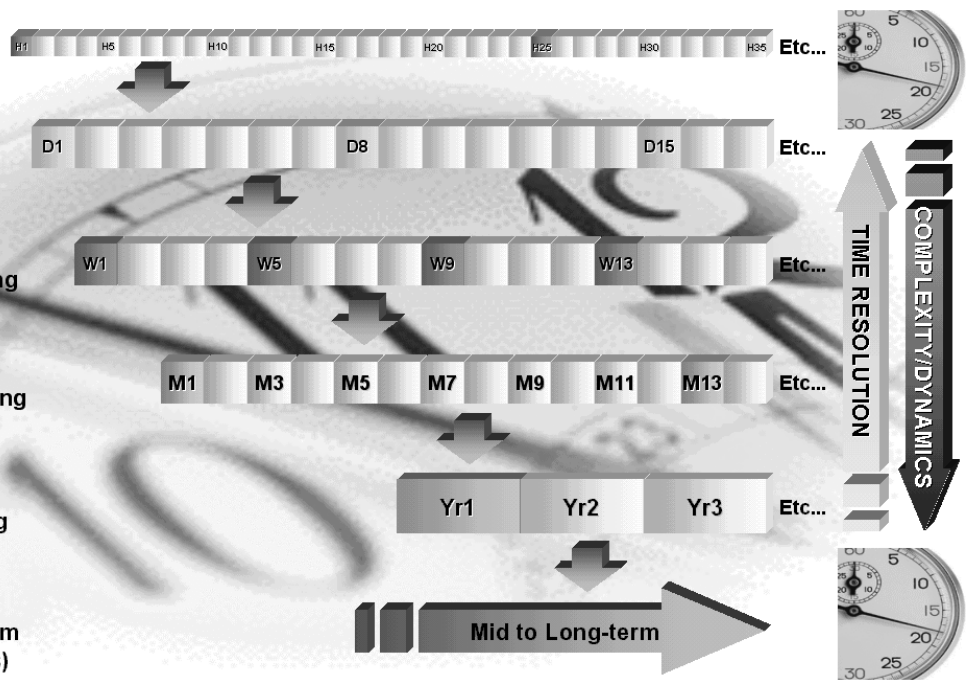

Fig. 1. EMCAS time scales and decision levels

\section{Electricity Markets Complex Adaptive Systems (EMCAS)}

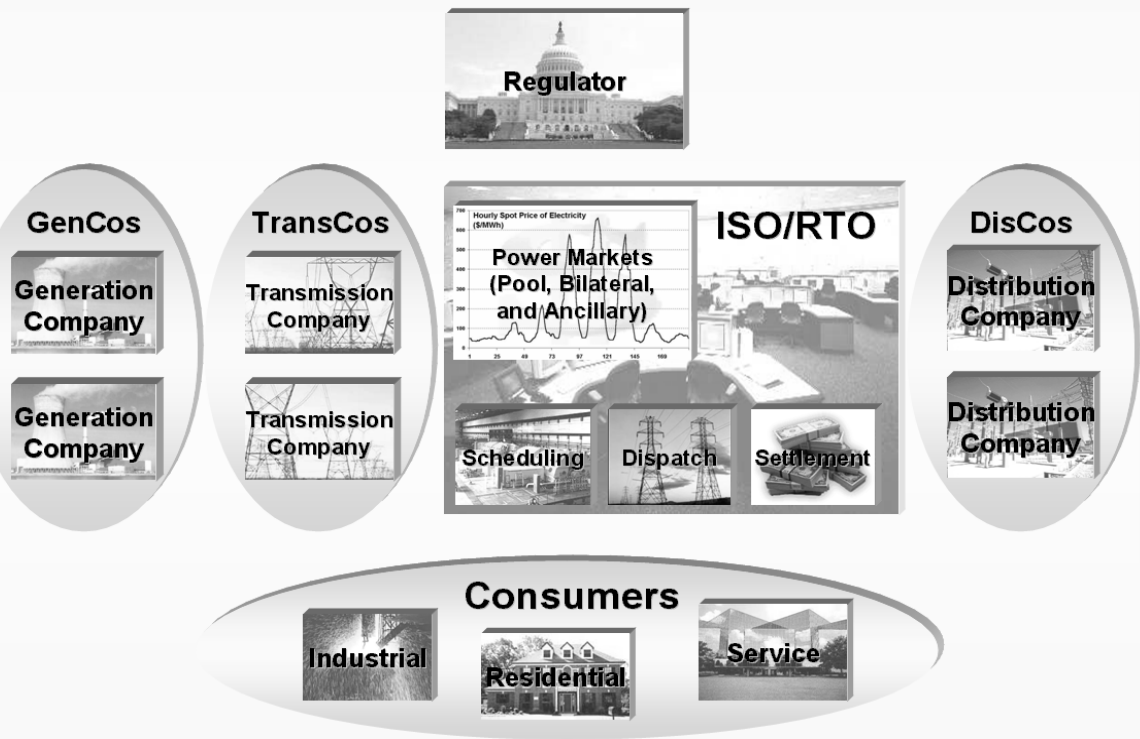

Fig. 2. EMCAS structure and agents 
The EMCAS interface client uses Dynamic Hypertext Markup Language (DHTML) and Scalable Vector Graphics (SVG), allowing it to be displayed in all major web browsers. The interface client can be used anywhere in the world that a server is available via the Internet or on portable computers without a network connection but with a local server.

One agent class used in the EMCAS modeling system is designed to simulate the behavior of Generating Company Agents (GCAs) and the marketing strategies that emerge as GCAs strive to exploit the physical limitations of the power system using the market rules under which they operate, as shown in Figure 3. GCAs can sell products in various markets. In EMCAS, a GCA learns the extent to which local and regional prices are influenced by its marketing strategies. This learning process is based on an "explore and exploit" process. Agents explore various marketing and bidding strategies. Once a strategy is found that performs well, it is exercised (i.e., exploited) and fine-tuned as subtle changes occur in the marketplace. When more dramatic market changes take place and a strategy begins to fail, an agent more frequently explores new strategies in an attempt to adapt to the dynamic and evolving supply-and-demand forces in the marketplace. Even when a strategy continues to perform well, a GCA periodically explores and evaluates other strategies in its search for one that performs better. However, the exploration rate tends to be significantly lower than under stressful conditions.

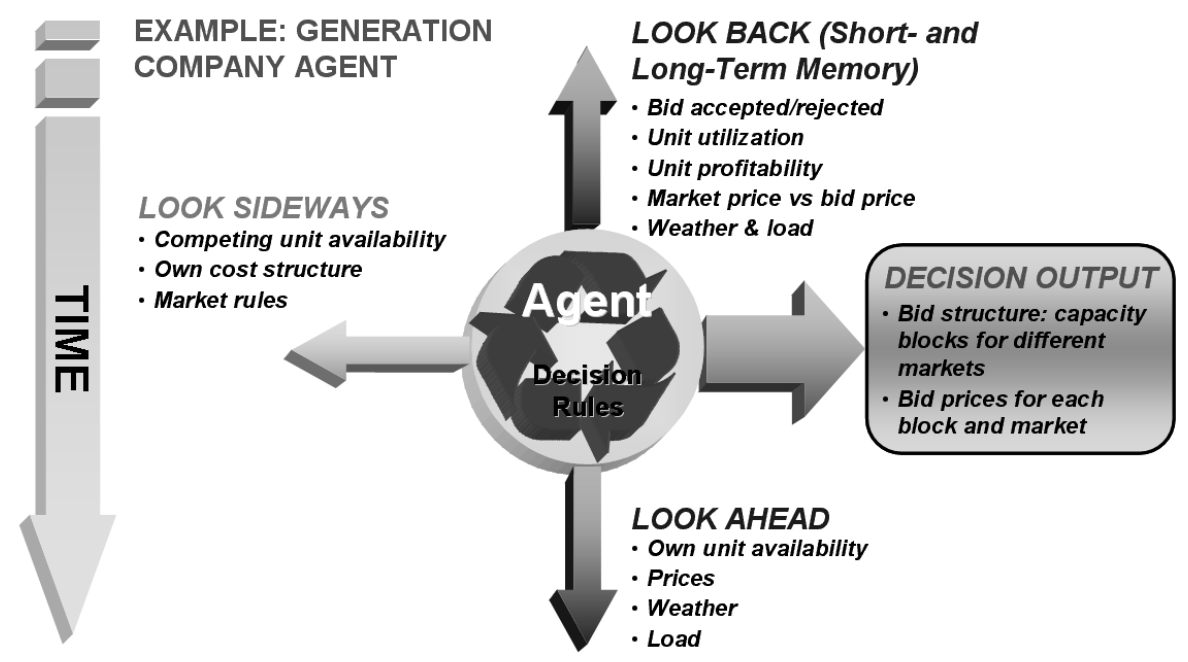

Fig. 3. Generation company agent

In EMCAS, a GCA is comprised of a number of building blocks that represent different tasks or actions an agent can perform. Each GCA seeks to arrange and parameterize these building blocks in a way that allows the market player to maximize its corporate utility. A building block consists of a set of one or more relatively simple rules. For example, one very simple agent rule may be if the GCAs sets the marketing clearing price in the last bidding period, then the GCA bid price in the next period will be fractionally higher." One parameter in this building block specifies the rate of change in the bid price. Although the basic building blocks are available to all GCAs, an ex- 
ploited strategy may not utilize a building block if it is discovered that it is not beneficial. However, if market conditions change or if the GCA discovers a new way to combine the building block with another one, it can be used to develop a new strategy. When a GCA owns and operates more than one generating unit, an integrated strategy is formulated, and the combined effects of unit-level actions are important. This may entail losing money at one facility to gain more profits at another one.

We propose that this same agent building block approach can be used to develop elements of a PSE. In particular, the approach can be adapted to address automatic ontology construction/extension; personalization; and real-time visual representation of both the program state and the "object of interest" state within a PSE.

\section{Automatic Ontology Construction and Extension}

An EMCAS agent makes decisions based on past experiences and anticipated conditions in the future and in the context of current market rules and the potential impact that other players will have on the markets. In the same way, a PSE environment can be described within a particular domain. The possible/reasonable paths through the PSE can then be broken down into directed graphs of discrete steps, or building blocks, each corresponding to a function/action that allows the user to progress towards the goal of "solving" the problem. Analytical agents can then be constructed from the discrete steps in response to goals set by the user via the user interface. As the problem or analysis is worked through, the agent reevaluates its context at each step, assembling the necessary blocks as required. An ontology constructed this way can then be saved for further use in later PSE applications. Since, in use, each step is accompanied by an evaluation of the blocks needed for the next step(s), the ontology is extended by simply using the PSE. We have found it possible to build arbitrarily complex behavior paths using this approach.

\subsection{Personalization}

By providing a user interface that allows each user to enter their preferences, this initial personalization becomes part of the PSE environment that the agents automatically use to assemble the problem solving paths that are to be used. As the user continues to use the PSE, the agents learn preferred paths, or tool use, by the continual assessment of the internal PSE environment.

\subsection{Real-Time Visual Representations within PSE}

True flexibility in a user input-and-display environment can be achieved by having functions delegate these functions to other services. This can be achieved for most functions, but is perhaps most easily discussed/illustrated for the case of real-time visual representation. Meta-protocols have been developed that transparently link domain objects. This allows domain objects to publish available data and functionality at run time, and allows changes in the state of domain objects to be displayed as they occur.

Domain objects include both those responsible for functional behavior in the PSE and those objects that are the subject of the analysis - usually data objects of some 
kind. The user can therefore watch a display of the PSE elements evolving during use, improving their understanding of the analysis process, e.g. which solver, lookup table, data set, etc., is being used. At the same time, the change in state of each actual agent in use and the values that the agent represents can also be displayed.

\section{Conclusion}

ABMs allow the use of complex adaptive systems approaches that are capable of producing tools or PSEs that can address the behavior of each of the participants within complex systems. In this paper, we argued that the same type of agent-based technology that is used to produce "realistic" agent behavior in EMCAS and other agentbased simulation tools at ANL can also be used to embed these tools in a PSE-type environment.

\section{References}

1. Gallopoulos, S., Houstis, E., Rice, J., Computer as Thinker/Doer: Problem-Solving Environments for Computational Science, IEEE Computational Science and Engineering, IEEE, 11-23 Vol. 1, No. 2: Summer 1994.

2. Bonabeau, E., Dorigo, M., Theraulaz, G., Swarm Intelligence: From Natural to Artificial Systems, Oxford University Press: 1999.

3. VanKuiken, J.C., Veselka, T.D., Guziel, K.A., Blodgett, D.W., Hamilton, S., Kavicky, J.A., Koritarov, V.S., North, M.J., Novickas, A.A., Paprockas, K.R., Portante, E.C., Willing, D.L., APEX User's Guide (Argonne Production, Expansion, and Exchange Model for Electrical Systems) Version 3.0, Argonne National Laboratory: 1994.

4. Veselka, T.D., Portante, E.C., Koritarov, V.S., Hamilton, S., VanKuiken, J.C., Paprockas, K.R., North, M.J., Kavicky, J.A., Guziel, K.A.., Poch, L.A., Folga, S., Tompkins, M.M., Novickas, A.A., Impacts of Western Area Power Administration's Power Marketing Alternatives on Electric Utility Systems, Argonne National Laboratory: 1994

5. North, M., Koritarov, V., Boyd, G., Veselka, T.D., Macal, C.M., Conzelmann, G.C. Thimmapuram, P.R., E-Laboratories: Agent-Based Modeling of Electricity Markets," American Power Conference, Electronic Proceedings, PennWell Corporation: April 2002 